\title{
EVOLUTIONARY CONCEPT BASED ON PALEONTOLOGICAL EVIDENCE
}

\author{
KRASSILOV, Valentin A., Paleontological Institute, 123 Profsojusnaya \\ Street, Moscow, 117647, Russia
}

Paleontology can provide a main source of predictive evolutionary theory rather than procede in its traditional role of a testing ground for theories based on a nonhistorical material. Fossil record testifies to a general increase of the global ecosystem efficiency through time. Insofar as ecosystem is essentially a life producing machine, its efficiency can be measured by the biomass to dead mass production ratio which is the lowest in the Proterozoic microbial ecosystems. The increased efficiency is due to division of labor reflected in the initial biological diversity of photosynthetic, heteroprophic, sedentary and vagile organisms representing the nearly simultaneously appearing basic life form types classified as the higher taxa of pro- and eukaryotes. In the interdepending - coherent - evolution each newly appearing form opens potential niches for more forms of both the lower and higher trophic levels in turn impelling a further increase of biological diversity. Upper limit to this self-accelerated process marked by diversity peaks of the fossil record is set by the minimal effective population size which depends on environmental stability: in unstable environments populations require a redundant frequency buffering them from occasional devastating impacts and, therefore, the diversity limit should be lower than in relatively stable environments. This model gives a long-sought explanation of the observed diversity stability correlation. In the coherently evolving communities competition decreases with the narrowing niche overlap while new species entries are due primarily to their innovative - new niche opening - capacities rather than to their competitive superiority. Thus there is an innovative stimulus instigating an increase of structural complexity - a general biological progress.

The fossil record of progressive evolution is periodicallyinterrupted by major and minor crises - the reversions of evolutionary tendencies typically occurring at or close to stratigraphic levels of intense tectonic, eustatic and climatic events the concerted ation of which may be due to rotational triggering. The crises intervals, such as the K/T transition, are marked by the low diversity mono- or oligodominant communities of highly polymorphic and prodigally prolific populations. The increased population redundancy is combined with the rising dead mass production evidenced by massive accumulation of organic-rich sediments. Ecological successions are truncated at their pioneer stages driving the respective climax species to mass extinctins. Suddenly appearing morphological novelties are of pedomorphic or chimeric nature (e.g., in Mesozoic proangiosperms) and are due to developmental acceleration related to proletarian reproductive strategy (e.g., in ecotonal areas and rift zones of the early angiosperm appearance). Their extended evolutionary potentials are to be realized in the next coherent stage. 